\title{
Hydroxychloroquine Inhibits Cardiac Conduction in Aged Patients with Nonmalaria Diseases
}

\author{
Yanting $\mathrm{Yu}^{\mathrm{a}}$ Jianteng $\mathrm{Xu}^{\mathrm{b}} \quad$ Anni Xie $^{\mathrm{a}}$ Sijia Liu $^{\mathrm{a}}$ Xiaojian Wang $^{\mathrm{a}}$ \\ Runzhang Zhu ${ }^{a}$ Xiaoyan Wang ${ }^{\text {a }}$ \\ aDepartment of Nephrology, Nanjing BenQ Medical Center, The Affiliated BenQ Hospital of Nanjing \\ Medical University, Nanjing, China; ' Department of Clinical Laboratory, Nanjing BenQ Medical Center, \\ The Affiliated BenQ Hospital of Nanjing Medical University, Nanjing, China
}

\section{Keywords}

Cardiac conduction - Electrocardiogram .

Hydroxychloroquine · Coronavirus disease 2019

\begin{abstract}
Background: The COVID-19 pandemic has brought increased focus on hydroxychloroquine (HCQ), as doctors, the medical community, and policymakers around the world attempt to understand how the risks of $\mathrm{HCQ}$ weigh against unknown benefits. We aim to evaluate the effects of $\mathrm{HCQ}$ on cardiac conduction, thus contributing to the global understanding of implications of HCQ use. Methods: We reviewed 717 cases of nonmalaria patients treated with HCQ (302) or without HCQ (415) in our hospital from 2008 to 2019, analyzed the cardiac conduction recorded by electrocardiogram (122 vs. 180) including heart rate (HR), PR, and corrected-QT (QTc) intervals, and explored the relationship of cardiac conduction with age, HCQ dosage, HCQ duration, sex, and primary diseases in HCQ users. Results: The all-cause mortality is similar between HCQ and non-HCQ groups ( 4.0 vs. $4.3 \%$, $p=0.85$ ). Patients aged 45 years or older, not younger ones,
\end{abstract}

karger@karger.com www.karger.com/kdd

Karger $\stackrel{\text { ' }}{5}$
(C) 2021 The Author(s)

Published by S. Karger AG, Basel

This is an Open Access article licensed under the Creative Common Attribution-NonCommercial-4.0 International License (CC BY-NC) (http://www.karger.com/Services/OpenAccessLicense), applicable to the online version of the article only. Usage and distribution for commercial purposes requires written permission. have lower HR ( $80.1 \pm 1.7$ vs. $85.7 \pm 1.8 \mathrm{bpm}, p=0.03$ ) but longer PR ( $163 \pm 3.4$ vs. $146.6 \pm 4.2 \mathrm{~ms}, p=0.003)$ and QTc $(417.8 \pm 3.8$ vs. $407.7 \pm 2.7 \mathrm{~ms}, p=0.03)$ in HCQ than those in non-HCQ. The age in the HCQ group is positively correlated with PR $(R=0.31, p<0.01)$ and QTc $(R=0.34, p<0.01)$ but not HR. HR, PR, and QTc are not related to HCQ dosage (0.1$0.6 \mathrm{~g} /$ day), $\mathrm{HCQ}$ duration (0.2-126 months), sex, primary diseases, and repeated exams. Conclusion: Age is the most important risk factor of $\mathrm{HCQ}$ on cardiac conduction in nonmalaria patients. Electrocardiogram monitoring is suggested in aged patients due to the effects of $H C Q$ on $H R, P R$, and QTc.

(c) 2021 The Author(s)

Published by S. Karger AG, Basel

\section{Introduction}

Hydroxychloroquine (HCQ), as a traditional antimalarial drug, was first synthesized in 1944 and approved by the US FDA in 1955 [1]. HCQ is developed by the addition of a $\beta$-hydroxy chain to the chloroquine (CQ) molecule, the first antimalarial drug, and has reduced toxicity but conserved efficacy compared to CQ. Both CQ and 
HCQ are reported in the management of nonmalaria diseases including systemic lupus erythematosus (SLE) [2], rheumatoid arthritis (RA) [3], Sjögren's syndrome (SS) [4], and others. Their potential beneficial effects have been shown in the cardiovascular system [5], hematological system [6], malignant diseases [7], and viral infections $[8,9]$. They accumulate preferentially in the acidic environment of lysosomes, phagolysosomes, and endosomes, stabilize the membranes of those organelles by raising $\mathrm{pH}$, and protect the tissues from inflammation injuries $[10,11]$.

The COVID-19 (coronavirus disease) pandemic has brought increased focus on CQ and HCQ as doctors, the medical community, and policymakers around the world attempt to understand how the drug risks weigh against unknown benefits [12]. Most recently, CQ or HCQ has been applied in the treatment of COVID-19 due to severe acute respiratory syndrome coronavirus 2 (SARS-CoV-2) infection [13]. They block the infectivity of the SARS$\mathrm{CoV}-2$ in vitro by interfering with virus-cell fusion and glycosylation of cellular receptors of SARS-CoV $[10,11]$ and are used to treat COVID-19 patients in different countries [14-17].

The side effects of HCQ include gastrointestinal disturbance [18], ocular toxicity [19], and cardiovascular complications [20]. Conduction disorders, especially QTc interval prolongation, may be fatal [21, 22]. A number of reports have shown that HCQ with or without azithromycin is associated with QT prolongation in the treatment of COVID-19 [23, 24]. Further studies are needed to determine the risk factors of HCQ usage in cardiac conduction among age, sex, pre-existing diseases, and dosage and duration of HCQ. In order to contribute to the global understanding of implications of HCQ use, we evaluated effects of HCQ on cardiac conduction by reviewing the hospitalized nonmalaria patients who were prescribed HCQ relative to the age-, sex-, and diseasematched ones who were not prescribed HCQ.

\section{Materials and Methods}

\section{Study Patients}

The study protocol was approved by the Institutional Review Board and Medical Ethics Committee of Nanjing BenQ Medical Center, The Affiliated BenQ Hospital of Nanjing Medical University (Approval No. 2020-KL008-01). This is a retrospective and observational study. All procedures performed in studies involving human participants were in accordance with the ethical standards of the institutional and/or national research committee and with the 1964 Helsinki Declaration and its later amendments or comparable ethical standards.
Using the Hospital Information System, the patients who were hospitalized and taking HCQ meanwhile in the Affiliated BenQ Hospital of Nanjing Medical University from May 12, 2008, to May 12,2019 , were considered as the HCQ group (302 cases). The patients who were diagnosed with the similar primary diseases but not taking HCQ were grouped into non-HCQ control (415 cases). All patients without a general agreement on follow-up inquires and data publication at admission were excluded. To match the distribution of primary diseases of the HCQ group, all cases diagnosed as SLE, RA, and SS were included while cases were randomly chosen (simple random sampling) from the system for dermatitis, dermatomyositis, erythroderma, eczema, vasculitis, other connective tissue diseases, and other diseases. The patients with antiarrhythmic drug treatment (e.g., amiodarone, flecainide, or sotalol) were excluded. Patients with electrocardiogram (ECG) records were analyzed for the cardiac conduction in the HCQ group (122 cases) and the non-HCQ group (180 cases). Patients in the HCQ group with repeated ECG and echocardiography (18 cases) were included to analyze the changes of cardiac conduction, cardiac function, and structure.

\section{Data Collection}

The hospital record numbers were provided by the IT staff based on HCQ usage or disease diagnosis. Anonymous information without patient's personal identification, address, telephone number, and email account was analyzed by professionals who had written agreements on patient's privacy protection.

The status of patient survival or all-cause death was obtained by the Hospital Information System or telephone inquiries from April 5, 2020, to May 12, 2020. The diagnosis of the primary diseases and comorbid diseases was collected from the discharging summary of the patients. Their sex, age, dosage and duration of HCQ, ECG records, and echocardiography records were collected.

\section{Resting ECG Examination}

A standard digitally recorded 12-lead resting supine ECG was performed by using an autoanalyzer (page writer trim III; Philips) automatically to record heart rate ( $\mathrm{HR}$, beats per minutes, bpm), $\mathrm{PR}$ interval (the time from the beginning of the $\mathrm{P}$ wave to the beginning of the QRS wave, ranged $0.12-0.20 \mathrm{~s}$ ), and QT interval (the interval from the beginning of the QRS wave to the end of the T wave). The corrected QT interval (QTc) was calculated as QT/ $\left(\mathrm{RR}^{0.5}\right)$. RR was calculated as 60 divided by the actual heart rate. The normal QTc interval is below $470 \mathrm{~ms}$ in males and $450 \mathrm{~ms}$ in females [25].

\section{Echocardiography Examination}

Philips Hdii Color Doppler ultrasound diagnostic instrument was used for echocardiography examination. The parameters of the cardiac function included left ventricular ejection fraction (EF, $\%)$. The parameters of the cardiac structure included left atrial diameter (LAD, mm), left ventricular diameter (LVD, $\mathrm{mm}$ ), and interventricular septum (IVS) thickness ( $\mathrm{mm})$.

\section{Relevant Medication}

Relevant medication that could impact cardiovascular conduction included azithromycin, quinolone antibiotics (such as levofloxacin and moxifloxacin), beta-blockers, glucocorticosteroids, thyroid hormone, nonsteroidal anti-inflammatory drugs, antipsychotics, and immunosuppressive drugs. 
Table 1. Demographic and clinical characteristics of all patients with or without HCQ

\begin{tabular}{lccc}
\hline & $\begin{array}{l}\text { HCQ } \\
(n=302)\end{array}$ & $\begin{array}{l}\text { Non-HCQ } \\
(n=415)\end{array}$ & $p$ value \\
\hline Female, $n(\%)$ & $207(68.5)$ & $265(63.9)$ & 0.20 \\
Age, years & $53.4 \pm 3.7$ & $55.8 \pm 2.3$ & 0.31 \\
Primary disease, $n(\%)$ & & & 0.13 \\
SLE & $69(22.9)$ & $75(18.1)$ & 0.36 \\
RA & $53(17.6)$ & $62(14.9)$ & 0.34 \\
SS & $39(12.9)$ & $43(10.4)$ & 0.58 \\
Dermatitis & $38(12.6)$ & $59(14.2)$ & 0.58 \\
Dermatomyositis & $27(8.9)$ & $32(7.7)$ & 0.06 \\
Connective tissue diseases & $19(6.3)$ & $43(10.4)$ & 0.56 \\
Erythroderma & $10(3.3)$ & $18(4.3)$ & 0.12 \\
Eczema & $10(3.3)$ & $25(6.0)$ & 0.77 \\
Vasculitis & $4(1.3)$ & $7(1.7)$ & 0.64 \\
Others & $33(10.9)$ & $51(12.3)$ & 0.4 \\
Comorbid disease, $n$ (\%) & & & 0.12 \\
Coronary atherosclerotic heart disease & $13(4.3)$ & $25(6)$ & 0.56 \\
Heart failure & $11(3.6)$ & $26(6.3)$ & 0.54 \\
Stroke & $10(3.3)$ & $18(4.3)$ & 0.79 \\
Hypertension & $35(11.6)$ & $42(10.1)$ & 0.15 \\
Diabetes & $29(9.6)$ & $37(8.9)$ & 0.8 \\
Chronic kidney disease & $65(21.5)$ & $71(17.1)$ & 0.85 \\
Cancer & $8(2.6)$ & $9(2.2)$ & \\
All-cause mortality & $12(4.0)$ & & \\
Events, $n$ (\%) & & & \\
\hline
\end{tabular}

All patients were followed up for 12-144 months, and the survival or death was recorded. HCQ, hydroxychloroquine; SLE, systemic lupus erythematosus; RA, rheumatoid arthritis; SS, Sjögren's syndrome.

Statistical Analysis

The results were expressed as the mean \pm SE. Student $t$ test was used for comparison between the 2 groups, and one-way ANOVA with LSD post hoc tests was used for comparison among 3 groups and above. Categorical variables were expressed as case number and ratio (\%), the latter was compared by the $\chi^{2}$ test. Pearson's correlation was used for correlations of age with cardiac conduction values. Statistical analyses were performed with SPSS version 23.0 (SPSS Inc., Chicago, IL, USA); $p<0.05$ was considered statistically significant. Figures were generated with GraphPad Prism 8.0 (GraphPad Software Inc., San Diego, CA, USA).

\section{Results}

All-Cause Mortality with or without HCQ Exposure

In the current study, there were 302 patients taking HCQ (HCQ group) and 415 patients not taking HCQ (non-HCQ group). There was no difference between the 2 groups in age, sex, ratio of primary diseases, and ratio of comorbid diseases, suggesting a similar background for group comparison. The all-cause mortality was simi- lar between the 2 groups ( 4.0 vs. $4.3 \%, p>0.05)$ with follow-up from 12 to 144 months (shown in Table 1). The duration of taking HCQ was from 0.2 to 126 months, with an average of $35.3 \pm 32.4$ months and a median of 35 months; the dosage was $0.1-0.6 \mathrm{~g} / \mathrm{day}$, with an average of $0.3 \pm 0.1 \mathrm{~g} /$ day and a median of $0.2 \mathrm{~g} /$ day.

\section{Cardiac Conduction of ECG with or without HCQ Exposure}

One-hundred twenty-two cases in HCQ and 180 cases in non-HCQ had ECG records. There was no difference between groups in age, sex, ratio of primary diseases, and ratio of combined medication that might affect the cardiac conduction (shown in Table 2).

HCQ patients had lower HR $(81.9 \pm 1.5$ vs. $87.1 \pm 1.6$ bpm, $p=0.03)$ but longer PR (159.3 \pm 2.8 vs. $146.1 \pm 3.3$ $\mathrm{ms}, p=0.002)$ than non-HCQ ones while the difference in QTc was not significant $(410.4 \pm 3.3$ vs. $405.9 \pm 2.3 \mathrm{~ms}$, $p=0.25$ ). In order to clarify the HCQ effects distinguished by age, the patients with similar distribution in age, sex, and primary diseases were divided into young groups 
Table 2. General information of the patients with ECG

\begin{tabular}{lccc}
\hline & $\begin{array}{l}\text { HCQ } \\
(n=122)\end{array}$ & $\begin{array}{l}\text { Non-HCQ } \\
(n=180)\end{array}$ & $p$ value \\
\hline Female, $n(\%)$ & $86(70.4)$ & $121(67.2)$ & 0.61 \\
Age, years & $54.5 \pm 1.6$ & $56.9 \pm 1.3$ & 0.27 \\
Primary disease, $n(\%)$ & & & \\
SLE & $28(22.9)$ & $38(21.1)$ & 0.78 \\
RA & $25(20.5)$ & $35(19.4)$ & 0.88 \\
SS & $23(18.9)$ & $30(16.7)$ & 0.65 \\
Dermatitis & $9(7.4)$ & $12(6.7)$ & 0.82 \\
Dermatomyositis & $6(4.9)$ & $17(9.4)$ & 0.18 \\
Connective tissue diseases & $10(8.2)$ & $16(8.9)$ & 1.0 \\
Comorbid disease, $n$ (\%) & $5(4.1)$ & $12(6.7)$ & 0.45 \\
Coronary atherosclerotic heart disease & $6(4.9)$ & $9(5)$ & 1.0 \\
Heart failure & $4(3.3)$ & $7(3.9)$ & 1.0 \\
Atrioventricular block & $3(2.5)$ & $7(3.9)$ & 0.75 \\
Right bundle-branch block & $6(4.9)$ & $9(5)$ & 1.0 \\
Hyperkalemia & $7(5.7)$ & $6(3.3)$ & 0.39 \\
Hypokalemia & & & 0.75 \\
Combined medication, $n$ (\%) & $3(2.5)$ & $71(3.9)$ & 0.50 \\
Azithromycin & $32(26.2)$ & $46(25.6)$ & 0.12 \\
Quinolone antibiotics & $42(34.4)$ & $98(54.4)$ & 1.00 \\
Beta-blockers & $67(54.9)$ & $12(6.7)$ & 0.63 \\
Glucocorticosteroids & $6(4.9)$ & $16(8.9)$ & 0.56 \\
Thyroid hormone & $14(11.5)$ & $18(10.0)$ & 0.06 \\
NSAID & $5(18)$ & $89(49.4)$ & 0.08 \\
Antipsychotic drugs & $22(59.8)$ & & \\
Immunosuppressive drugs & & & \\
\hline
\end{tabular}

ECG, electrocardiogram; HCQ, hydroxychloroquine; NSAID, nonsteroidal anti-inflammatory drug; SLE, systemic lupus erythematosus; RA, rheumatoid arthritis; SS, Sjögren's syndrome.

( $<45$ years old, 32 vs. 43 cases in HCQ and non-HCQ groups) and aged groups ( $\geq 45$ years old, 90 vs. 137 cases in HCQ and non-HCQ), respectively, for further analyses. For the patients in the aged groups, HR was lower $(80.1 \pm 1.7$ vs. $85.7 \pm 1.8 \mathrm{bpm}, p=0.03)$, but $\mathrm{PR}(163 \pm 3.4$ vs. $146.6 \pm 4.2 \mathrm{~ms}, p=0.003)$ and QTc $(417.8 \pm 3.8$ vs. $407.7 \pm 2.7 \mathrm{~ms}, p=0.03)$ were longer in HCQ than nonHCQ; HR, PR, and QTc were similar between HCQ and non-HCQ groups in the young patients (Student's $t$ test, $p>0.05$ ) (shown in Fig. 1).

Two cases in the HCQ group and 1 case in the nonHCQ group met the diagnostic criteria of QTc prolongation. In the HCQ group, one (light eruption patient, QTc: $482 \mathrm{~ms}$ ) was given pacemaker installation while the other (SS patient, QTc: $489 \mathrm{~ms}$ ) was asymptomatic (shown in online suppl. Fig. 1; for all online suppl. material, see www.karger.com/doi/10.1159/000515278). In the nonHCQ group, one eczema patient had chest tightness (QTc: $508 \mathrm{~ms}$ ). All 3 patients were above 70 years and still alive until the data were collected.
The Age with Cardiac Conduction in HCQ Patients

The age in the HCQ group was positively correlated with PR $(r=0.31, p<0.001)$ and QTc $(r=0.34, p<0.001)$ but not $\mathrm{HR}$, indicating that cardiac conduction was prolonged along with the increase of age (Pearson's correlation) (shown in Fig. 2); low but significant correlation of age with PR and QTc was also found in non-HCQ patients (shown in online suppl. Fig. 2). These findings suggested a synergetic effect of age with HCQ on cardiac conduction.

\section{The Dosage and Duration of HCQ with Cardiac Conduction in HCQ Exposure}

According to the daily dosage of HCQ, 122 patients with ECG were divided into 2 groups including $\leq 0.2 \mathrm{~g} /$ day (64 cases) and $>0.2 \mathrm{~g} /$ day (58 cases). There was no difference in HR $(79.9 \pm 1.9$ vs. $84.2 \pm 2.5)$, PR (163.1 \pm 4.5 vs. $155.3 \pm 3.1)$, and QTc $(409.1 \pm 4.3$ vs. $411.8 \pm 5.1)$ between the 2 groups (Student $t$ test, $p>0.05$ ) (shown in Fig. 3a). 


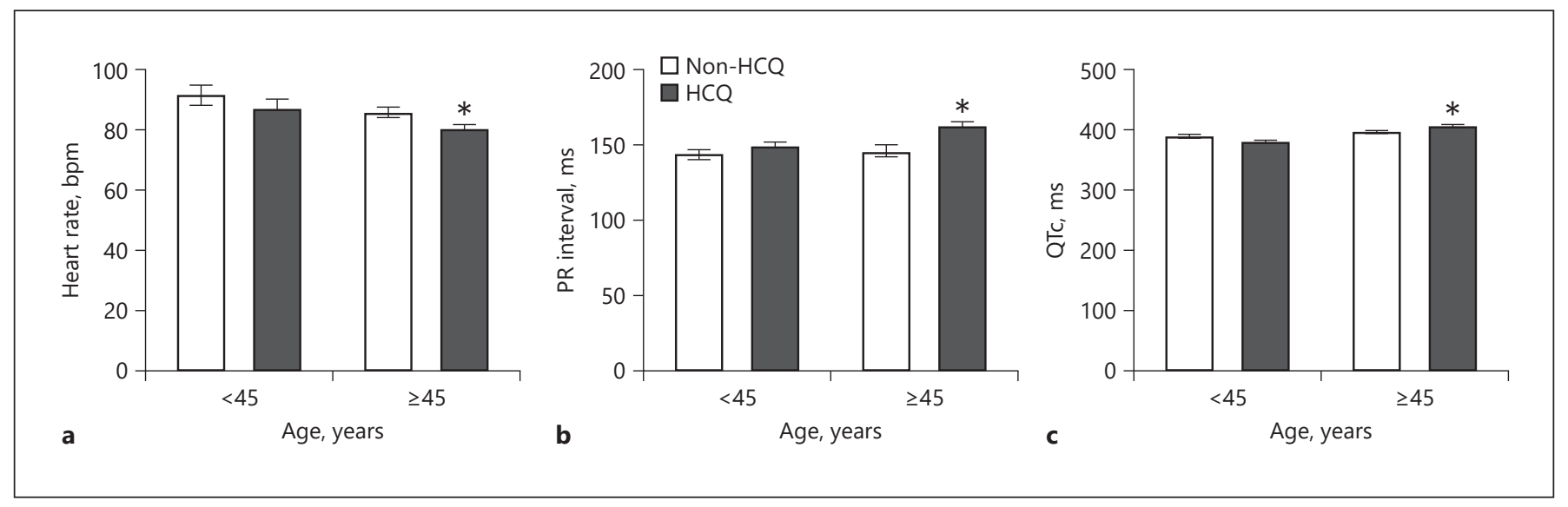

Fig. 1. Analyses of HCQ usage with cardiac conduction in young and old patients between HCQ and non-HCQ groups. One-hundred twenty-two patients in the HCQ group and 180 patients in the non-HCQ group, who were diagnosed with similar diseases, were divided into the young group ( $<45$ years old, 32 vs. 43 cases) and the old group ( $\geq 45$ years old, 90 vs. 137 cases), respectively. The cardiac conduction with ECG was analyzed (Student's $t$ test,
${ }^{*} p<0.05$ vs. non-HCQ group). a Comparison of HR between HCQ and non-HCQ groups. b Comparison of PR between HCQ and non-HCQ groups. c Comparison of QTc between HCQ and nonHCQ groups; QTc calculated by QT/(RR $\left.{ }^{\wedge} 0.5\right)$. RR was calculated as 60 divided by the actual heart rate (bpm). HCQ, hydroxychloroquine; ECG, electrocardiogram; HR, heart rate; PR, PR intervals; QTc, corrected-QT intervals.

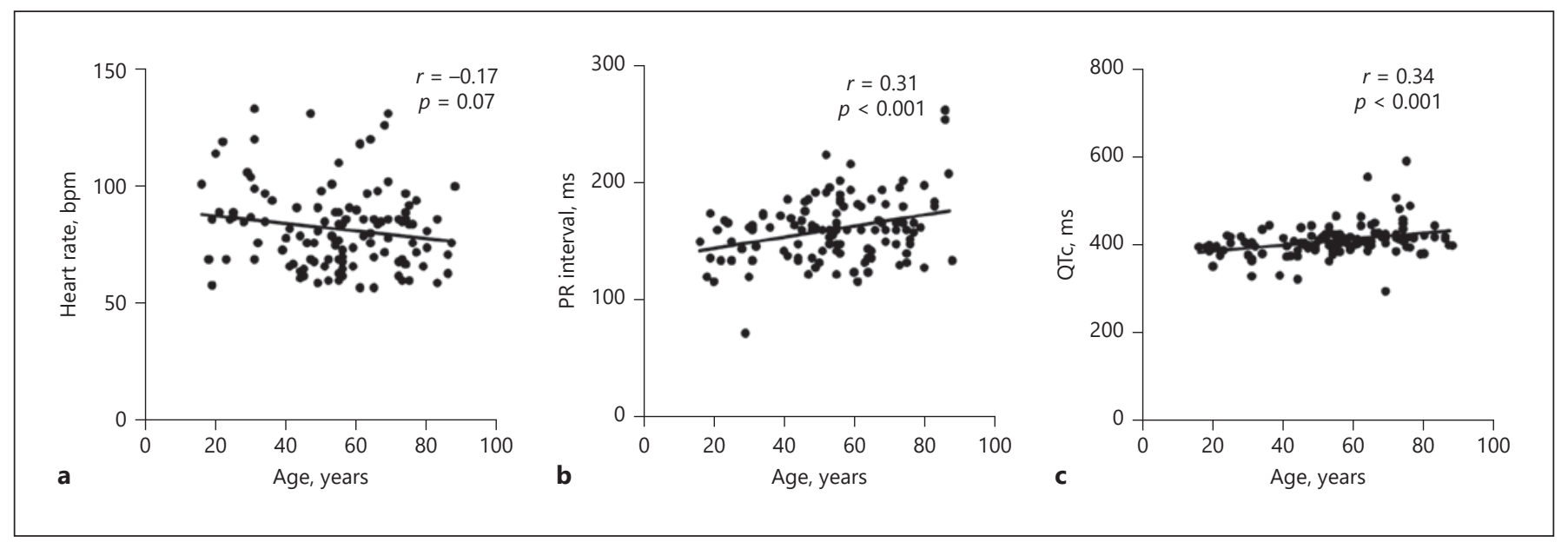

Fig. 2. Correlation analyses of age with cardiac conduction in HCQ patients. One-hundred twenty-two patients, who were prescribed HCQ, were included in the analyses (Pearson's correlation). a The correlation of age with $\operatorname{HR}(r=-0.17, p=0.07)$. $\mathbf{b}$ The correlation of age with $\mathrm{PR}(r=0.31, p<0.001)$. c The correlation of age with QTc $(r=0.34, p<0.001)$. HCQ, hydroxychloroquine; HR, heart rate; PR, PR intervals; QTc, corrected-QT intervals.
One-hundred twenty-two patients with ECG were divided into 4 groups based on HCQ duration: $<12$ months ( 26 cases), $12-36$ months ( 58 cases), $36-60$ months ( 24 cases), and $>60$ months ( 14 cases), respectively. There were no differences in the HR $(80.4 \pm 17.6,79.6 \pm 16.8,85.9 \pm 16.5$, and $87.7 \pm 17.6 \mathrm{bpm}, p>0.05)$, PR interval $(159.4 \pm 21.2$, $161.7 \pm 29.2,161.8 \pm 24.7$, and $156.3 \pm 34.8 \mathrm{~ms}, p>0.05)$, and QTc $(399.3 \pm 31.8,415.8 \pm 35.2,410.5 \pm 44.2$, and $408.4 \pm 33.9 \mathrm{~ms}, p>0.05)$ among groups (one-way ANOVA, LSD test, all $p>0.05$ ) (shown in Fig. 3b).

\section{Sex and Primary Diseases with Cardiac Conduction in HCQ Exposure}

There was no difference in HR $(81.2 \pm 16.4$ vs. $79.5 \pm$ $19.1 \mathrm{bpm}, p>0.05)$, PR interval (168.8 \pm 27.6 vs. $164.9 \pm$ $35.7 \mathrm{~ms}, p>0.05)$, and QTc interval (408.7 \pm 28.3 vs. 


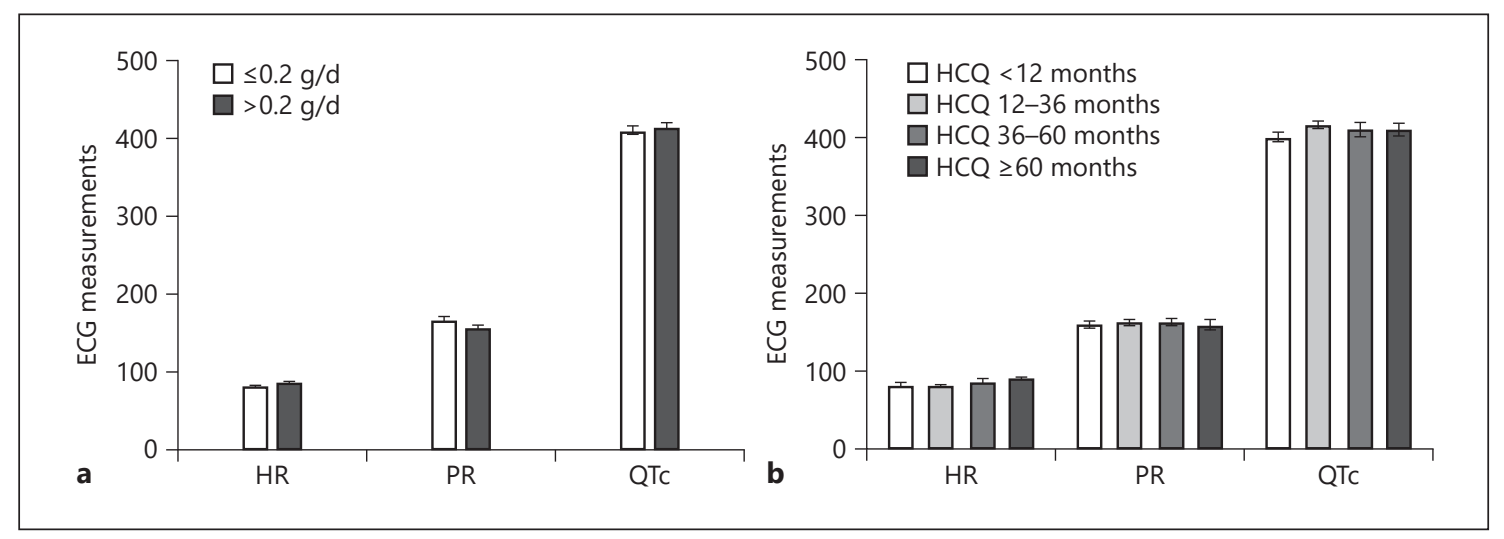

Fig. 3. Analyses of the cardiac conduction with HCQ dosage and HCQ duration. One-hundred twenty-two patients, who were prescribed HCQ, had ECG and were included. a Cardiac conduction with HCQ dosage. The patients were divided into 2 groups including $\leq 0.2 \mathrm{~g} /$ day ( 64 cases) and $>0.2 \mathrm{~g} /$ day ( 58 cases) according to the daily dosage of HCQ (Student $t$ test, $p>0.05$ ). b Cardiac conduc- tion with HCQ duration. One-hundred twenty-two patients were divided into 4 groups: < 12 months ( 26 cases), $12-36$ months (58 cases), 36-60 months ( 24 cases), and $\geq 60$ months ( 14 cases), according to the HCQ duration (one-way ANOVA, LSD test, all $p>$ 0.05). HCQ, hydroxychloroquine; ECG, electrocardiogram; HR, heart rate; PR, PR intervals; QTc, corrected-QT intervals.

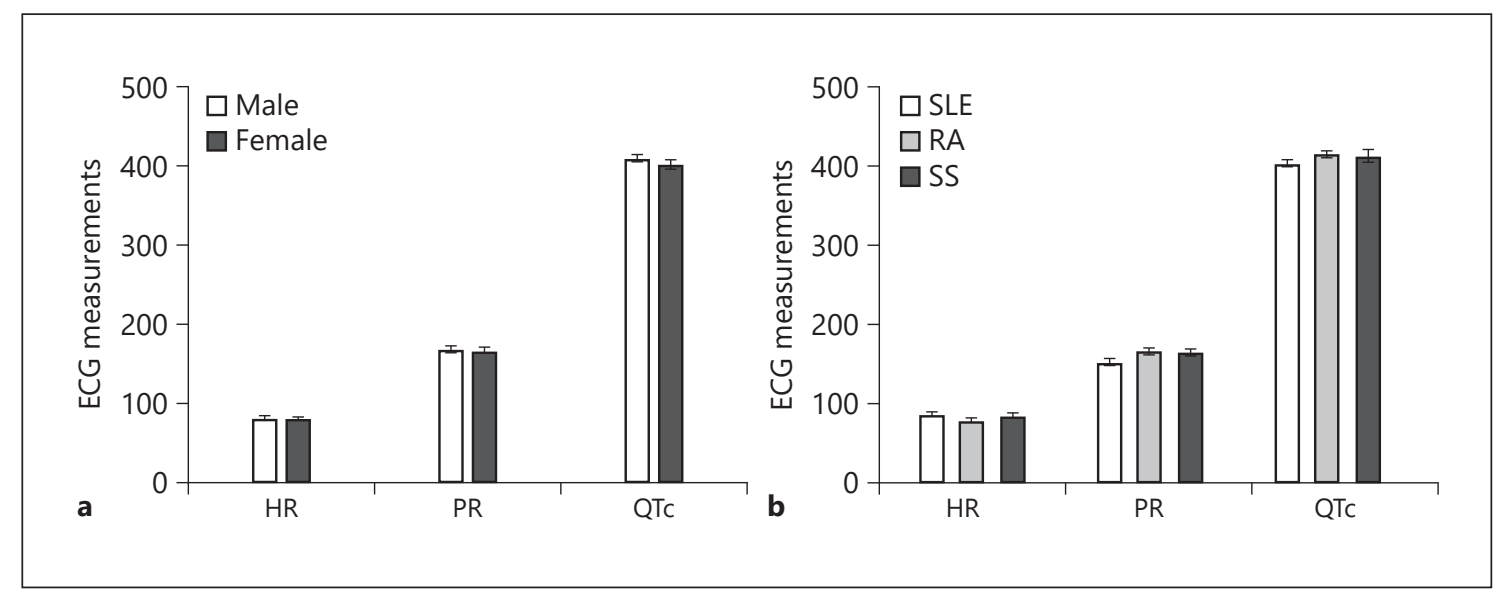

Fig. 4. Analyses of the cardiac conduction with sex and primary diseases. One-hundred twenty-two patients, who were prescribed HCQ, had ECG and were included. a Cardiac conduction with the sex of HCQ patients. HCQ patients were divided into male (36 cases) and female (86 cases) groups (one-way ANOVA, LSD test, all $p>0.05)$. $\mathbf{b}$ Cardiac conduction with the diagnosis of primary

$398.7 \pm 67.6 \mathrm{~ms}, p>0.05)$ between males ( 36 cases) and females (86 cases) (Student's $t$ test) (shown in Fig. 4a). Among the top 3 primary diseases including SLE (28cases), RA (25cases), and SS (23cases), no significant difference was found in HR $(85.5 \pm 18.2,78.9 \pm 14.2$, and $82.7 \pm 18.7 \mathrm{bpm})$, PR interval $(152.2 \pm 23.9,166 \pm 19.4$, and $163.3 \pm 25 \mathrm{~ms})$, and QTc interval $(400.4 \pm 24.2$, $414 \pm 21.3$, and $409.7 \pm 47.3 \mathrm{~ms}$ ) (one-way ANOVA, LSD test, all $p>0.05$ ) (shown in Fig. $4 b$ ). diseases. The top 3 diseases in HCQ patients were analyzed including SLE (28 cases), RA ( 25 cases), and SS (23 cases) (one-way ANOVA, LSD test, all $p>0.05$ ). HCQ, hydroxychloroquine; ECG, electrocardiogram; HR, heart rate; PR, PR intervals; QTc, corrected-QT intervals; SLE, systemic lupus erythematosus; RA, rheumatoid arthritis; SS, Sjögren's syndrome.

\section{Repeated Examinations of ECG and \\ Echocardiography in HCQ Exposure}

Eighteen over 122 patients in the HCQ group received repeated ECG examination with the gap from 1 to 38 months, median for 6 months. There was no significant difference in HR $(74.8 \pm 10.7$ vs. $76.6 \pm 13.8 \mathrm{bpm}, p>$ $0.05)$, PR interval (165.1 \pm 15.5 vs. $162.5 \pm 15.7 \mathrm{~ms}, p>$ $0.05)$, and QTc interval ( $412.4 \pm 25.1$ vs. $417.6 \pm 53.7 \mathrm{~ms}$, $p>0.05$ ) between the first and second ECG (shown in 


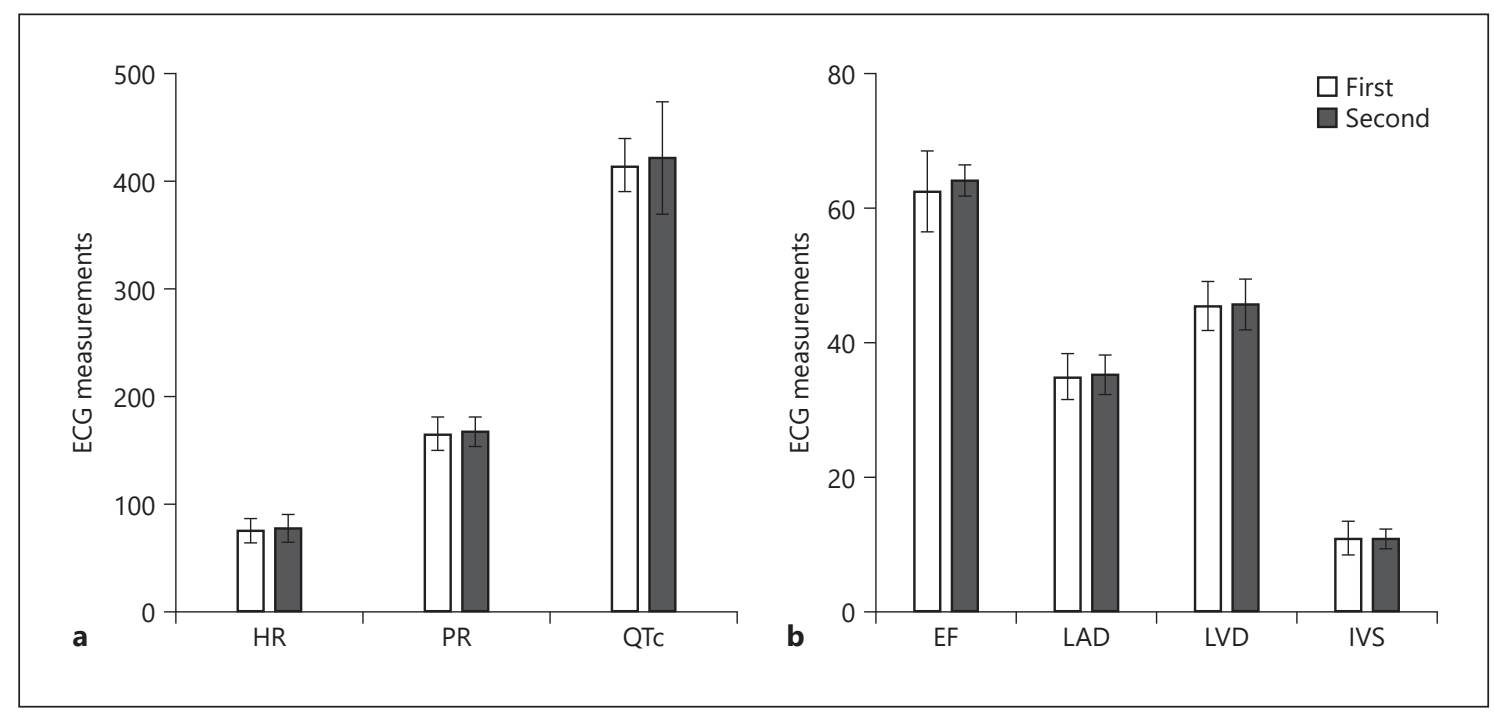

Fig. 5. The first and second cardiac parameters on ECG and echocardiography. Eighteen over 122 patients, who were prescribed HCQ, received repeated examination of ECG and color Doppler echocardiography. a The cardiac conduction parameters (HR, PR, and QTc) were compared between the first and second ECG (Student's $t$ test, all $p>0.05$ ) with the gap from 1 to 38 months, median for 6 months. $\mathbf{b}$ The cardiac function (EF) and structural parame-

Fig. 5a). The 18 patients also received repeated echocardiography with the gap from 3 to 41 months, median for 9 months. $\mathrm{EF}(62.6 \pm 6$ vs. $64 \pm 2.4, p>0.05)$, LAD $(34.9 \pm 3.8$ vs. $34.4 \pm 3.3 \mathrm{~mm}, p>0.05), \operatorname{LVD}(45.4 \pm 3.8$ vs. $45.4 \pm 4.0 \mathrm{~mm}, p>0.05)$, and IVS thickness $(10.9 \pm 2.5$ vs. $10.3 \pm 1.8 \mathrm{~mm}, p>0.05)$ were also similar between the 2 exams (Student's $t$ test) (shown in Fig. 5b).

\section{Discussion}

The safety of HCQ, especially for cardiac conduction, in the COVID-19 is controversial. In this study, we analyzed the all-cause mortality of patients taking HCQ in relative to those not taking HCQ in our hospital for 11 years. We found that the mortality of all causes in 2 groups was similarly low (about 4\%) in the follow-up period of 1-12 years. Further detailed analyses of the effects of HCQ on cardiac conduction were based on the ECG records of HR, PR interval, and QTc interval. For patients aged 45 years or older, HR was lower but PR and QTc were longer in HCQ than non-HCQ while there was no difference between HCQ and non-HCQ for patients below 45 years old (shown in Fig. 1a, b). The age was positively correlated with PR and QTc, not HR, in the HCQ ters (LAD, LVD, and IVS) were compared between the first and second echocardiography (Student's $t$ test, all $p>0.05$ ) with the gap ranged from 3 to 41 months, a median of 9 months. HCQ, hydroxychloroquine; ECG, electrocardiogram; HR, heart rate; PR, PR intervals; QTc, corrected-QT intervals; EF, left ventricular ejection fraction (\%); LAD, left atrial diameter (mm); LVD, left ventricular diameter (mm); IVS, interventricular septum thickness ( $\mathrm{mm}$ ). group (shown in Fig. 2b, c), suggesting that PR and QTc of HCQ patients were prolonged along with the increase of age. Two cases in the HCQ group and 1 case in the nonHCQ group diagnosed with QTc prolongation were all above 70 years old. HR, PR, and QTc were not altered with dosage (0.1-0.6 g/day) and duration of HCQ (0.2126 months). The cardiac conduction was not changed with sex and primary diseases. These findings indicate that age is the most important risk factor of HCQ on cardiac conduction in nonmalaria patients. ECG monitoring is suggested in aged HCQ users due to the effects of HCQ on HR, PR, and QTc. The differences between HCQ and non-HCQ are small but may be of clinical significance in application regime for disease control using HCQ, especially in the prevention and treatment of COVID-19 pandemic.

HCQ has been recognized as a safe medicine generally, even for pregnancy [26]. A cardiovascular protection effect of CQ/CQ is reported in several nonmalaria diseases. Fardet and colleagues [27] have reported that in incident cancer patients, the risk of death is lower in the ones chronically exposed to HCQ/CQ compared with those unexposed in the overall population. Sharma and coworkers [28] have showed that chronic HCQ exposure is associated with a $72 \%$ decrease in the risk of incident car- 
diovascular disease in RA patients. Exposure to HCQ/CQ before the diagnosis of lupus nephritis was negatively associated with the development of hypertension and thrombosis [29]. On the contrary, long-term HCQ appears to have no vascular protective effect in patients with SLE [30]. Among COVID-19 patients hospitalized in New York, treatment with HCQ, azithromycin, or both, compared with neither treatment, is not significantly associated with differences in in-hospital mortality. Our results show that there was no difference in the all-cause mortality between HCQ group and non-HCQ group, consistent with the systematic review of antimalarial drugs [31, 32].

HCQ causes cardiac conduction disorder, especially QTc interval prolongation, in various reports [21]. In patients with COVID-19 treated with HCQ/azithromycin, 58 of 251 patients (23\%), at least 1 measure of extreme QTc interval prolongation was observed. And, 35 of 58 patients $(60 \%)$ who were diagnosed QTc interval prolongation were not on any other QTc-prolonging medication [24]. It is also reported that ECG abnormalities have no association with HCQ usage [33]. A recent nested case-control study has showed that HCQ/CQ decreases the odds of ECG conduction abnormalities in 453 SLE patients [34]. It has been suggested that cardiotoxicity may be enhanced by older age, pre-existing cardiac disease, and renal insufficiency $[35,36]$. In the current report, HR was decreased but PR interval and QTc interval were increased in HCQ patients relative to non-HCQ ones with age older than 45 years while there was no difference in HR, PR, and QTc for patients younger than 45 years. Furthermore, the age was positively correlated with PR and QTc. Though the incidence of QTc prolongation is high in COVID-19 patients treated with HCQ/azithromycin, reported in Chorin's study [24], the mean age was $64 \pm 13$ years, and the age of baseline QRS duration $\geq 120$ ms was $73 \pm 9$ years, where both were older than 45 years. Our findings indicate that age is a critical factor of HCQ on cardiac conduction abnormalities, which may explain the confliction of the reports in general.

The exact dosages with cardiac toxicity of HCQ/CQ are not well defined. Recently, research has found evidence of ventricular arrhythmia in 2 COVID-19 patients from a group of 28 treated with high-dose CQ [37]. Ursing et al. [38] have showed that high-dose CQ for uncomplicated Plasmodium falciparum malaria is well tolerated and causes QT interval prolongation similar to standard-dose CQ in children. Chen et al. [21] have reported QT interval prolongation with refractory ventricular arrhythmia in a patient on HCQ $0.2 \mathrm{~g} /$ day treatment for 1 year. However,
McGhie et al. [34] have found that HCQ and CQ cumulative dose above the median $(1,207 \mathrm{~g})$ decreases the odds of ECG conduction abnormalities in 453 SLE patients. In our study, the HCQ dosage 0.1-0.6 g/day was not correlated with any changes in HR, PR interval, and QTc interval. Costedoat-Chalumeau and coworkers [39] have reported that the duration of antimalarial use varies widely in patients with cardiac toxicity, ranging from 3 months to 27 years. In another report, the duration of HCQ/CQ use longer than 5 years is not a statistically significant predictor of either cardiac conduction disorders or structural abnormalities [34]. In this study, the duration of HCQ varied from 0.2 to 126 months, and there was no difference in HR, $\mathrm{PR}$, and QTc among 4 groups with various HCQ durations, consistent with McGhie et al. [34]. In addition, we did not find any difference in HR, PR, and QTc between sex and among the top 3 primary diseases for HCQ patients.

Cardiac structural abnormalities are less common than conduction abnormalities in HCQ/CQ-induced cardiotoxicity. Scientists [34] have reported that antimalaria cumulative dose is not associated with cardiac structural abnormalities (left ventricular hypertrophy or atrial enlargement), but SLE duration and eGFR were statistically significantly associated with structural ECG abnormalities. However, HCQ-induced cardiomyopathy is linked to limited cutaneous systemic sclerosis [40] and SLE [20, 41]. In this study, we did not find any statistical difference in cardiac structure between the first and second examination including LAD, LVD, IVS, and cardiac function (EF\%) with taking HCQ. However, the interpretation of these findings may be subject to limitation in case number included in the analyses.

\section{Conclusion}

Taking HCQ due to various diseases does not increase the all-cause mortality in our patients. Aging accelerates the HCQ-induced prolongation of PR and QTc intervals. The cardiac conduction is not related to duration and dosage of HCQ, sex, and primary diseases. Therefore, ECG monitoring is suggested for aged HCQ users. HCQ up to $0.6 \mathrm{~g} /$ day might be used in patients younger than 45 years old without affecting QTc.

\section{Acknowledgements}

The authors thank the patients for the follow-up information provided and Suhua Wang, Head of Information Technology department in our hospital, for the list of patients included. 


\section{Statement of Ethics}

The study was approved by the Institutional Review Board and Medical Ethics Committee of Nanjing BenQ Medical Center, The Affiliated BenQ Hospital of Nanjing Medical University (Approval No. 2020-KL008-01). Due to the retrospective nature of the study, written informed consent for participation in the study was waived.

\section{Conflict of Interest Statement}

The authors declare no competing interests.

\section{Funding Sources}

This work was supported by grants from the Nanjing Science and Technology Development Foundation (201715065), Natural Science Foundation of China (81900650 and 81970605), and Natural Science Foundation of Jiangsu Province (BK20190128).

\section{Author Contributions}

All authors contributed to the study. Y.Y. collected and assembled the data and wrote the manuscript. X.A., L.S., W.X.J., and Z.R. collected and assembled the data. X.J. did data analysis and interpretation. W.X.Y. conceptualized and designed the study, revised and approved the final version of the submitted manuscript, and is responsible for the integrity of all data.

\section{References}

1 Wallace DJ. The history of antimalarials. Lupus. 1996 Jun;5(Suppl 1):S2-3.

2 Fanouriakis A, Kostopoulou M, Alunno A, Aringer M, Bajema I, Boletis JN, et al. 2019 update of the EULAR recommendations for the management of systemic lupus erythematosus. Ann Rheum Dis. 2019 Jun;78(6):73645.

3 Kyburz D, Brentano F, Gay S. Mode of action of hydroxychloroquine in RA-evidence of an inhibitory effect on toll-like receptor signaling. Nat Clin Pract Rheumatol. 2006 Sep;2(9): 458-9.

4 Ben-Zvi I, Kivity S, Langevitz P, Shoenfeld Y. Hydroxychloroquine: from malaria to autoimmunity. Clin Rev Allergy Immunol. 2012 Apr;42(2):145-53.

5 Rainsford KD, Parke AL, Clifford-Rashotte M, Kean WF. Therapy and pharmacological properties of hydroxychloroquine and chloroquine in treatment of systemic lupus erythematosus, rheumatoid arthritis and related diseases. Inflammopharmacology. 2015 Oct; 23(5):231-69.

6 Espinola RG, Pierangeli SS, Gharavi AE, Harris EN, Ghara AE. Hydroxychloroquine reverses platelet activation induced by human IgG antiphospholipid antibodies. Thromb Haemost. 2002 Mar;87(3):518-22.

7 Sasaki K, Tsuno NH, Sunami E, Tsurita G, Kawai K, Okaji Y, et al. Chloroquine potentiates the anti-cancer effect of 5-fluorouracil on colon cancer cells. BMC Cancer. 2010 Jul 15; 10:370.

8 Keyaerts E, Vijgen L, Maes P, Neyts J, Van Ranst M. In vitro inhibition of severe acute respiratory syndrome coronavirus by chloroquine. Biochem Biophys Res Commun. 2004 Oct 8;323(1):264-8.

9 Paton NI, Aboulhab J. hydroxyurea and didanosine as initial therapy for HIV-infected patients with low viral load: safety, efficacy and resistance profile after 144 weeks. HIV Med. 2005 Jan;6(1):13-20.
10 Colson P, Rolain JM, Lagier JC, Brouqui P, Raoult D. Chloroquine and hydroxychloroquine as available weapons to fight $\mathrm{CO}$ VID-19. Int J Antimicrob Agents. 2020 Apr; 55(4):105932.

11 Wang M, Cao R, Zhang L, Yang X, Liu J, Xu $\mathrm{M}$, et al. Remdesivir and chloroquine effectively inhibit the recently emerged novel coronavirus (2019-nCoV) in vitro. Cell Res. 2020 Mar;30(3):269-71.

12 Qian JY, Wang B, Liu BC. Acute Kidney Injury in the 2019 novel coronavirus disease. Kidney Dis. 2020 Jun;323:1-6.

13 Huang C, Wang Y, Li X, Ren L, Zhao J, Hu Y, et al. Clinical features of patients infected with 2019 novel coronavirus in Wuhan, China. Lancet. 2020 Feb 15;395(10223):497-506.

14 Gautret P, Lagier JC, Parola P, Hoang VT, Meddeb L, Mailhe M, et al. Hydroxychloroquine and azithromycin as a treatment of $\mathrm{CO}$ VID-19: results of an open-label non-randomized clinical trial. Int $J$ Antimicrob Agents. 2020 Mar 20;56(1):105949.

15 Huang M, Tang T, Pang P, Li M, Ma R, Lu J, et al. Treating COVID-19 with Chloroquine. J Mol Cell Biol. 2020 May 18;12(4):322-5.

16 Risch HA. Early outpatient treatment of symptomatic, high-risk COVID-19 patients that should be ramped up immediately as key to the pandemic crisis. Am J Epidemiol. 2020 Nov 2;189(11):1218-26.

17 Sylaja PN, Srivastava MVP, Shah S, Bhatia R, Khurana D, Sharma A, et al. The SARSCoV-2/COVID-19 pandemic and challenges in stroke care in India. Ann N Y Acad Sci. 2020 Aug;1473(1):3-10.

18 Tett S, Cutler D, Day R. Antimalarials in rheumatic diseases. Baillieres Clin Rheumatol. 1990 Dec;4(3):467-89.

19 Jones SK. Ocular toxicity and hydroxychloroquine: guidelines for screening. Br J Dermatol. 1999 Jan;140(1):3-7.
20 Tselios K, Gladman DD, Harvey P, Mak S, Chantal M, Butany J, et al. Hydroxychloroquine-Induced Cardiomyopathy in Systemic Lupus Erythematosus. J Clin Rheumatol. 2016 Aug;22(5):287-8.

21 Chen CY, Wang FL, Lin CC. Chronic hydroxychloroquine use associated with QT prolongation and refractory ventricular arrhythmia. Clin Toxicol (Phila). 2006;44(2): 173-5.

22 O'Laughlin JP, Mehta PH, Wong BC. Life threatening severe QTc prolongation in patient with systemic lupus erythematosus due to hydroxychloroquine. Case Rep Cardiol. 2016;2016:4626279.

23 Costedoat-Chalumeau N, Hulot JS, Amoura Z, Leroux G, Lechat P, Funck-Brentano C, et al. Heart conduction disorders related to antimalarials toxicity: an analysis of electrocardiograms in 85 patients treated with hydroxychloroquine for connective tissue diseases. Rheumatology. 2007 May; 46(5): 808-10.

24 Chorin E, Wadhwani L, Magnani S, Dai M, Shulman E, Nadeau-Routhier C, et al. QT interval prolongation and torsade de pointes in patients with COVID-19 treated with hydroxychloroquine/azithromycin. Heart Rhythm. 2020 Sep;17(9):1425-33.

25 Crotti L, Celano G, Dagradi F, Schwartz PJ. Congenital long QT syndrome. Orphanet J Rare Dis. 2008 Jul 7;3:18.

26 Gerosa M, Schioppo T, Meroni PL. Challenges and treatment options for rheumatoid arthritis during pregnancy. Expert Opin Pharmacother. 2016 Aug;17(11):1539-47.

27 Fardet L, Nazareth I, Petersen I. Effects of chronic exposure of hydroxychloroquine/ chloroquine on the risk of cancer, metastasis, and death: a population-based cohort study on patients with connective tissue diseases. Clin Epidemiol. 2017;9:545-54. 
28 Sharma TS, Wasko MC, Tang X, Vedamurthy D, Yan X, Cote J, et al. Hydroxychloroquine Use Is Associated With Decreased Incident Cardiovascular Events in Rheumatoid Arthritis Patients. J Am Heart Assoc. 2016 Jan 4; $5(1)$.

29 Siso A, Ramos-Casals M, Bove A, Brito-Zeron P, Soria N, Munoz S, et al. Previous antimalarial therapy in patients diagnosed with lupus nephritis: influence on outcomes and survival. Lupus. 2008 Apr;17(4):281-8.

30 Hsu CY, Lin YS, Su YJ, Lin HF, Lin MS, Syu YJ, et al. Effect of long-term hydroxychloroquine on vascular events in patients with systemic lupus erythematosus: a database prospective cohort study. Rheumatology. 2017 Dec 1;56(12):2212-21.

31 Haeusler IL, Chan XHS, Guérin PJ, White NJ. The arrhythmogenic cardiotoxicity of the quinoline and structurally related antimalarial drugs: a systematic review. BMC Med. 2018 Nov 7;16(1):200.

32 Rosenberg ES, Dufort EM, Udo T, Wilberschied LA, Kumar J, Tesoriero J, et al. Association of treatment with hydroxychloro- quine or azithromycin with in-hospital mortality in patients With COVID-19 in New York State. JAMA. 2020 Jun 23;323(24): 2493-502.

33 AlTwajery M, AlMane W, Al-Mayouf SM. Electrocardiographic disturbances in children with systemic lupus erythematosus. Int J Pediatr Adolesc Med. 2018 Dec;5(4):127-30.

34 McGhie TK, Harvey P, Su J, Anderson N, Tomlinson G, Touma Z. Electrocardiogram abnormalities related to anti-malarials in systemic lupus erythematosus. Clin Exp Rheumatol. 2018 Jul-Aug;36(4):545-51.

35 August C, Holzhausen HJ, Schmoldt A, Pompecki R, Schröder S. Histological and ultrastructural findings in chloroquine-induced cardiomyopathy. J Mol Med. 1995 Feb;73(2): 73-7.

36 Nord JE, Shah PK, Rinaldi RZ, Weisman MH. Hydroxychloroquine cardiotoxicity in systemic lupus erythematosus: a report of 2 cases and review of the literature. Semin Arthritis Rheum. 2004 Apr;33(5):336-51.

37 Jankelson L, Karam G, Becker ML, Chinitz LA, Tsai MC. QT prolongation, torsades de pointes, and sudden death with short courses of chloroquine or hydroxychloroquine as used in COVID-19: a systematic review. Heart Rhythm. 2020 Sep;17(9):1472-9.

38 Ursing J, Rombo L, Eksborg S, Larson L, Bruvoll A, Tarning J, et al. High-dose chloroquine for uncomplicated plasmodium falciparum malaria is well tolerated and causes similar qt interval prolongation as standard-dose chloroquine in children. Antimicrob Agents Chemother. 2020 Feb 21;64(3).

39 Costedoat-Chalumeau N, Hulot JS, Amoura Z, Delcourt A, Maisonobe T, Dorent R, et al. Cardiomyopathy related to antimalarial therapy with illustrative case report. Cardiology. 2007;107(2):73-80.

40 Abdin A, Pöss J, Kandolf R, Thiele H. Hydroxychloroquine-induced cardiomyopathy in a patient with limited cutaneous systemic sclerosis. Clin Res Cardiol. 2017 Mar;106(3): 234-6.

41 Zhao H, Wald J, Palmer M, Han Y. Hydroxychloroquine-induced cardiomyopathy and heart failure in twins. J Thorac Dis. 2018 Jan; 10(1):E70-3. 